\title{
Spectral Clustering of Psychological Networks
}

Michael Brusco, Florida State University, mbrusco@fsu.edu

Douglas Steinley, University of Missouri, steinleyd@ missouri.edu

Ashley L.Watts, University of Missouri, wattsal@missouri.edu

May 22, 2021 


\section{Spectral Clustering of Psychological Networks}

\section{Abstract}

Spectral clustering is a well-known method for clustering the vertices of an undirected network. Although its use in network psychometrics has been limited, spectral clustering has a close relationship to the commonly-used walktrap algorithm. In this paper, we report results from four simulation experiments designed to evaluate the ability of spectral clustering and the walktrap algorithm to recover underlying cluster structure in networks. The salient findings include: (1) the cluster recovery performance of the walktrap algorithm can be improved slightly by using exact $K$-means clustering instead of hierarchical clustering; (2) $K$-means and $K$-median clustering led to comparable recovery performance when used to cluster vertices based on the eigenvectors of Laplacian matrices in spectral clustering; (3) spectral clustering using the unnormalized Laplacian matrix generally yielded inferior cluster recovery in comparison to the other methods; (4) when the correct number of clusters was provided for the methods, spectral clustering using the normalized Laplacian matrix led to better recovery than the walktrap algorithm; (5) when the number of clusters was unknown, spectral clustering using the normalized Laplacian matrix was appreciably better than the walktrap algorithm when the clusters were equally-sized, but the two methods were competitive when the clusters were not equally-sized. Overall, both the walktrap algorithm and spectral clustering of the normalized Laplacian matrix are effective for partitioning the vertices of undirected networks, with the latter performing better in most instances.

\section{Keywords}

Network psychometrics, spectral clustering, $K$-means clustering, $K$-median clustering, walktrap algorithm. 
Research pertaining to the estimation and analysis of psychological networks has proliferated in the literature since the foundational work of Cramer et al. (2010a, 2010b). Procedures for estimating the edge weights of network matrices include $l_{1}$-regularized inverse covariance estimation (Epskamp \& Fried, 2018; Friedman et al., 2008, 2014), $l_{1}$-regularized logistic regression (van Borkulo et al., 2014), nonregularized partial correlation methods (Williams et al., 2019; Williams \& Rast, 2020), directed acyclic graphs (Scutari, 2010), and relative importance methods (Grömping, 2006). In this paper, we are not concerned with the process for establishing the edge weights for the psychological network. Instead, our focus is on a post hoc disentangling of the structural relationships between items based on the $p \times p$ edge-weight (adjacency) matrix corresponding to $p$ items that represent the vertices of the network graph, irrespective of the particular type of model and estimation process used to obtain that matrix.

There are different types of techniques that can be used for identifying these structural relationships. For example, Jones, Mair, and McNally (2018) recently surveyed a variety of visualization approaches, such as force-directed algorithms (Fruchterman \& Reingold, 1991), multidimensional scaling (Borg et al., 2018), and eigenmodels (Hoff et al., 2002). Matrix factorization methods have been described by Marsman et al. (2015, 2018). Brusco et al. (in press) demonstrated that matrix permutation methods could be used to produce sequences of items based on directional flow or similarity. Clustering methods provide another useful approach for discovering structure among items in network psychometrics and include the walktrap algorithm (Pons \& Latapy, 2006), the spin-glass algorithm (Reichardt \& Bornholdt, 2006), and clique percolation (Blanken et al., 2018; Palla et al., 2005). The walktrap algorithm, in particular, has been an especially popular clustering method in the recent network psychometrics literature (Briganti et al., 2018; Choi et al., 2017; Christensen, 2018; Fried, 2016; 
Golino \& Demetriou, 2017; Hevey, 2018; Jones, Mair, Riemann, et al. 2018; Kendler et al., 2018; McElroy \& Patalay, 2019). In addition, the exploratory graph analysis method developed by Golino and Epskamp (2017) uses the walktrap algorithm from igraph (Csardi \& Nepusz, 2006) as its community detection engine.

Pons and Latapy (2006) developed the walktrap algorithm as a method for detecting communities (dense subgraphs) in large (thousands of vertices) sparse graphs. The method uses a graph similarity measure based on random walks and, in this respect, is very similar to spectral clustering methods (Mohar, 1991, 1997; Shi \& Malik, 2000; von Luxburg, 2007) that are based on the eigendecomposition of the Laplacian matrix of the network graph. Pons and Latapy (2006, p. 198) note: “...the spectral approach takes an important part in the search for community structure in graphs", but cite the computational limitation of spectral clustering that pertains to the need to compute eigenvectors for large sparse graphs when there are thousands of vertices. They further observe that, relative to spectral clustering: "Our approach is based on the same foundation but has the advantage of avoiding the expensive computation of the eigenvectors" (Pons \& Latapy, 2006, p. 198). Succinctly, the walktrap algorithm is a computationally viable alternative to spectral clustering for large, sparse networks.

Most graphs in the network psychometrics literature are not large. Rather than thousands of vertices, the number of vertices in psychopathology applications is typically in the range of 10 to 100. The network graphs are also not sparse, in that the number of estimated edges is commonly at least $30 \%$ of the number of possible edges. In light of these properties of psychological networks, spectral clustering is computationally feasible and is a reasonable alternative to the walktrap algorithm. One of the key advantages of spectral clustering is that the eigenvectors of the Laplacian matrix are computed easily and it is not necessary to rely on an approximated 
similarity matrix based on transition probabilities. Another advantage is that the walktrap algorithm relies on a potentially suboptimal agglomerative hierarchical clustering approach based on Ward's (1963) method. Although the agglomerative hierarchical clustering algorithm is greedy, selecting the optimal merger of clusters at each stage, it is not guaranteed to produce a minimum sum-of-squares clustering for the selected number of clusters. For some of the smaller graphs encountered in network psychometrics, exact $K$-means clustering methods (i.e., methods guaranteed to provide a globally-optimal solution) can be used to cluster the items based on the relevant eigenvectors of the Laplacian matrix.

The use of spectral clustering is not without precedent in psychological research. For example, Chen et al. (2017) describe the use of the method for item classification within the context of personality assessment. The authors meticulously describe the process of constructing the similarity matrix based on Kendall's tau, $K$-nearest neighbors, and a connectivity threshold $(\tau)$, noting that it “... should be chosen such that the resulting graph $S$ is neither too sparse nor too dense" (Chen et al., 2017, p. 584). Fortunately, in network psychometrics, the graph estimation process often produces a network with a density of at least $30 \%$ and the use of connectivity thresholds is unnecessary.

Nevertheless, there are some important decisions associated with spectral clustering that require attention. The first decision is the selection of the form of the Laplacian matrix. The second decision is the selection of the number of clusters, which is often accomplished by seeking the largest 'gap' in the eigenvalues. A third decision is the method used to cluster the eigenvectors. Although $K$-means clustering (MacQueen, 1967; Steinhaus, 1956; Steinley, 2006) is typically recommended in the literature (Shi \& Malik, 2000; von Luxburg, 2007), $K$-median 
clustering (also known as p-median clustering; Brusco \& Köhn, 2008a, 2008b; Hakimi, 1964, 1965; Köhn et al., 2010) is also a viable alternative.

Our focus here is to assess the utility of spectral clustering and compare its performance to the walktrap algorithm for networks of the size and structure commonly observed in the network psychometrics literature. In the next section, we describe the walktrap algorithm and present the mathematical formulation of spectral clustering. This is followed by two motivating examples where we compare spectral clustering to results obtained via the walktrap algorithm. The first is a network pertaining to depressive symptoms from the clinical literature (Kendler et al., 2018), whereas the second network is from the social/personality literature and relates to attributes of Barack Obama (Dalege et al., 2017). Subsequently, we report the results of a series of simulation experiments designed to assess the ability of spectral clustering to recover a 'true' (or known) cluster structure under different network characteristics pertaining to the number of clusters, within-cluster cohesion, and cluster overlap. The simulation experiments facilitate comparisons of two different Laplacian matrices and both the $K$-median and $K$-means approaches for clustering the eigenvectors. In addition, two versions of the walktrap algorithm are evaluated in the comparative studies. The paper concludes with a summary and discussion of limitations and extensions.

\section{Methods}

Our description of the walktrap algorithm and spectral clustering methods for psychological networks begins with the definition of $V=\{1,2, \ldots p\}$ as a set of $p$ vertices that correspond to the items under study. We assume that a similarity relationship among the items is available in the form of a symmetric $p \times p$ adjacency matrix, $\mathbf{A}$, which contains nonnegative off-diagonal entries $a_{i j}=a_{j i}$. The edge-weight matrix can be obtained using methods for any of the general network 
types described by McNally (2016): (i) association networks, (ii) concentration networks, (iii) relative importance networks, and (iv) directed acyclic graphs. Regularized regression and logistic regression methods, which fall under the category of concentration network techniques, are perhaps the most common approaches for producing the edge-weight matrices. Although these methods can yield both positive and negative ties between items, most of the edge-weight matrices we have observed in the literature contain either exclusively positive elements, or mostly positive elements with a few small negative edge weights. For our purposes, we work with the absolute values of the edge weights because our primary interest is in whether ties do or do not exist. This is a common practice in the literature (Epskamp \& Fried, 2018) and nonnegative symmetric adjacency matrices are assumed for both spectral clustering and the walktrap algorithm.

\section{Walktrap algorithm}

The walktrap algorithm is a well-known and readily available method for community detection. However, as noted in the introduction, it was primarily designed for large, sparse networks that are not easily tackled using spectral decomposition. Nevertheless, the algorithm can be applied to the smaller networks encountered in network psychometrics. The algorithm begins with the computation of the degree matrix, $\mathbf{D}=\left[d_{i j}\right]$, which is a $p \times p$ diagonal matrix with main diagonal elements equal to the degree centrality measure for the items:

$$
d_{i i}=\sum_{j=1}^{p} a_{i j}
$$

The transition matrix, $\mathbf{P}=\mathbf{D}^{-1} \mathbf{A}$, contains elements $p_{i j}$ that represent the probability of a transition from vertex $i$ to vertex $j$ for a walk of length one. This can be generalized to a transition matrix for a walk of length $t$ via exponentiation of $\mathbf{P}$. More specifically, the matrix $\mathbf{P}^{t}$ contains elements $p_{i j}^{t}$ that represent the probability of a transition from vertex $i$ to vertex $j$ in $t$ steps. Next, a matrix 
of squared Euclidean distances between vertices is obtained based on their row vectors in $\mathbf{P}^{t}$ and a hierarchical clustering algorithm is subsequently used to produce a partition of the vertices.

There are several aspects of the walktrap algorithm that should be recognized by psychological researchers: (1) the assumption of loops (self edges), (2) the need to specify the edge weights for the loops in the case of weighted networks, (3) the need to select the length (number of steps) for the random walk, (4) the potential suboptimality of the hierarchical clustering algorithm, and (5) the determination of the number of clusters based on modularity.

Pons and Latapy (2006, p. 192) note: "To take into account the self-similarity of vertices, we impose that each vertex is linked to itself by a loop...". In the case of unweighted graphs, loops are incorporated by using a main diagonal of ones in the adjacency matrix. However, in the case of weighted adjacency matrices, it is not intuitively clear how the edge weights should be specified. For example, the average values of the off-diagonal elements for each row of the adjacency matrix could be used to establish the main diagonal. Alternatively, the maximum values within the rows could be used. Whatever the method used, it is important to understand that the partition of the vertices obtained can depend on the manner in which weights for the loops are determined.

The partition obtained by the walktrap algorithm is also sensitive to the number of steps selected for the random walk $(t)$. The selection of $t$ must be sufficiently large to provide adequate exploration of the network graph, yet not so large as to approach the steady state limits for the transition matrix (Fortunato \& Hric, 2016). In the limit, the columns of the transition matrix contain constants equal to the degree for the vertex divided by the sum of the degrees. Fortunately, small values for $t$ would seem to be sufficient for the smaller networks encountered 
in network psychometrics and two to five steps is a common range. A value of $t=4$ is the default for the implementation of the walktrap method in igraph.

For a given input matrix and walk length, it should be understood that the hierarchical clustering procedure used in the walktrap algorithm, which is based on Ward's (1963) method, is a greedy heuristic that does not necessarily produce the optimal partition for a given number of clusters. That is, for a given number of clusters, the resulting partition is not guaranteed to provide the minimum average squared distance between vertices and their clusters/communities (Pons \& Latapy, 2006, p. 201). For the smaller networks commonly encountered in network psychometrics, it will often be possible to use an exact $K$-means method based on branch-andbound programming to obtain a guaranteed optimal solution to the partitioning problem.

Finally, the determination of the number of clusters is a thorny issue for most (although not all) clustering methods. Pons and Latapy (2006, p. 203) propose the use of $Q$ modularity (Newman, 2004; Newman \& Girvan, 2004) for selecting the number of clusters when using the walktrap algorithm. The limits of this approach, particularly in the presence of small clusters, are well known (Hoffman et al., 2018; Lancichinetti \& Fortunato, 2011). Pons and Latapy also discuss the potential for using the following ratio rule: The change in the average squared distance between vertices and their clusters when going from $K+1$ to $K$ clusters divided by the corresponding change in average squared distance when going from $K$ to $K$-1 clusters. Similar rules have been shown to be effective in other clustering contexts (Brusco et al., 2017; Ceulemans \& Van Mechelen, 2005; Dimitriadou et al., 2002; Wilderjans et al., 2013).

We have implemented the walktrap algorithm in MATLAB (MATLAB, 2020) via an m-file, walktrap.m. The inputs to the algorithm are: (1) a nonnegative symmetric adjacency matrix, (2) the steps parameter, $t$, and (3) the number of clusters, $K$. If $K>0$, then the walktrap algorithm 
produces a partition of the vertices into $K$ clusters. The main diagonal elements of the adjacency matrix are established using the maximum edge-weights for each row. If $K=0$, then the algorithm determines and resets $K$ based on the ratio rule. A matrix of squared distances is obtained based on the transition matrix for $t$ steps and two partitions are generated. The first partition is produced using the hierarchical clustering approach, whereas the second is based on an optimal partition of the distance matrix using branch-and-bound programming (Brusco, 2006).

\section{Spectral clustering}

The unnormalized Laplacian matrix (Mohar, 1991, 1997) is $\mathbf{L}=\mathbf{D}-\mathbf{A}$. A popular normalized Laplacian matrix was proposed by Shi and Malik (2000). It is based on the random walk (see von Luxburg, 2007, pp. 404-405) and, accordingly, is closely related to the walktrap algorithm. Denoting, I as a $p \times p$ identity matrix, this random-walk Laplacian is:

$$
\mathbf{L}_{\mathrm{rW}}=\mathbf{D}^{-1} \mathbf{L}=\mathbf{I}-\mathbf{D}^{-1} \mathbf{A}
$$

In particular, it is noteworthy that the $\mathbf{D}^{-1} \mathbf{A}$ term in Equation (2) is the transition matrix for random walk processes that is the foundational component for the walktrap algorithm as described above (see Pons \& Latapy, 2006, p. 194).

With these definitions in place, the steps of the spectral clustering algorithm for the normalized graph Laplacian as proposed by Shi and Malik (2000) are as follows:

Step 0. Input A.

Step 1. Compute $\mathbf{D}$ and find the eigenvalues $\left(\lambda_{1}, \lambda_{2}, \ldots, \lambda_{p}\right)$ and eigenvectors $\left(\mathbf{u}_{1}, \mathbf{u}_{2}, \ldots, \mathbf{u}_{p}\right)$ of $\mathbf{L}_{\mathrm{rw}}$ by solving the generalized eigenproblem $\mathbf{L u}=\lambda \mathbf{D u}$. The eigenvalues and their corresponding eigenvectors are sequenced in increasing order of the eigenvalues.

Step 2. Choose the number of clusters, $K$. 
Step 3. Cluster the $p$ items based using the first $K$ eigenvectors as the clustering variables using $K$-means clustering. Let $\pi=\left\{C_{1}, \ldots, C_{K}\right\}$ represent the partition of the items into $K$ clusters where $C_{k}$ contains the items in cluster $k$ (for $1 \leq k \leq K$ ).

If the unnormalized Laplacian matrix is to be used, then Step 1 is modified to consist of a standard eigendecomposition of $\mathbf{L}$. The selection of the number of clusters in Step 2 can be specified by the user, or it can be determined using a variety of different criteria (Chen et al., 2017; von Luxburg, 2007). One of the most common approaches is the eigengap heuristic, where $K$ is chosen based on the largest gap in the increase of the eigenvalues over some range [ $K_{\min }$, $\left.K_{\max }\right]$. Specifically, we can compute $\lambda_{K}^{d}=\lambda_{K+1}-\lambda_{K}$ for $K_{\min } \leq K \leq K_{\max }$ and select the number of clusters based on the maximum value of $\lambda_{K}^{d}$. Although straightforward, von Luxburg (2007, p. 410) notes that the eigengap heuristic is well-suited for spectral clustering and is supported by results from perturbation theory and geometric aspects of spectral graph clustering (see, for example, Mohar, 1997). We evaluate the eigengap heuristic's ability to return the correct number of clusters in our Simulations 3 and 4 below. We have implemented spectral clustering in MATLAB (MATLAB, 2020) via an m-file, spectral.m.

$\boldsymbol{K}$-means clustering. The clustering of items in Step 3 is typically accomplished using $K$-means clustering. It is important at this juncture to distinguish between the $K$-means clustering optimization problem and $K$-means clustering algorithms. The $K$-means optimization problem is to find the partition of the $p$ items into $K$ clusters than minimizes the within-cluster sum-ofsquared deviation of the items from their cluster centroids (this criterion is commonly known as the $S S E$ ). The number of possible partitions of $p$ items into $K$ clusters is a Stirling number of the second kind (Brusco, 2006; Hand, 1981) and is computed as follows:

$$
\frac{1}{K !} \sum_{k=0}^{K}(-1)^{k}\left(\begin{array}{l}
K \\
k
\end{array}\right)(K-k)^{p} .
$$


The number of feasible partitions can be enormous even for relatively modest number of items. For example, for the $p=24$-item test problems in Section 4, there are more than $6.09 \times 10^{15}$ (i.e., more than 6.09 quadrillion) partitions of the items into $K=6$ clusters.

Because it is impossible to enumerate and evaluate all of the possible partitions for all but the smallest problems, the $K$-means algorithms that have been used in the literature are typically heuristic in nature. They are heuristics in the sense that they are not guaranteed to provide a partition that minimizes the within-cluster sum-of-squared deviations of items from their cluster centroids. The most popular heuristic algorithm has its origins in the work of Thorndike (1953) and Steinhaus (1956), and was later formalized by Forgy (1965) and MacQueen (1967). The algorithm begins with the specification of $K$ initial centroids (seed points). Next, each item is assigned to the cluster associated with its nearest centroid. The centroids are subsequently recomputed after this reassignment and the algorithm iterates between reassignment of items to clusters and recomputation of the centroids until no items change cluster membership. ${ }^{1}$ The algorithm is sensitive to the initial seed points and, therefore, it is recommended that it be restarted a large number of times (Steinley [2003] recommends 5000) using different initial seeds to assure that a poor local minimum is avoided. It should also be noted that running Ward's (1963) hierarchical clustering algorithm and cutting the tree at $K$ clusters is also a heuristic approach to the $K$-means clustering problem. ${ }^{2}$

Some algorithms for the $K$-means clustering optimization problem are known as exact methods because, unlike heuristics, they are guaranteed to provide a globally-optimal solution to the problem. One such algorithm was developed by Brusco (2006) and is based on the principles of branch-and-bound programming (Brusco \& Stahl, 2005; Hand, 1981). Branch-and-bound

\footnotetext{
${ }^{1}$ There are also some error-checking conditions that are typically incorporated to assure that no clusters are empty.

${ }^{2}$ Accordingly, the use of Ward's method in the walktrap algorithm should be viewed as heuristic in nature.
} 
algorithms are also referred to as implicit enumeration algorithms because they typically circumvent the need to explicitly evaluate all possible solutions. For example, suppose that we have an upper bound for the best possible value of the SSE for a $K$-means partitioning problem. In addition, assume that we have assigned some, but not all, of the $p$ items to clusters and compute a lower bound on the best possible SSE that could be realized from completing the assignment of the remaining items to clusters. If this lower bound exceeds the upper bound, then there is no need to enumerate and evaluate all of the possible solutions (branches) that could be realized from completing the solution. Thus, these possible solutions have not been explicitly evaluated but they have been implicitly discredited as possible optimal solutions.

In light of the modest size of most graphs in the network psychometrics literature, the branch-and-bound approach will often be feasible for solving the $K$-means optimization problem in Step 3 of spectral clustering. We use a MATLAB (MATLAB, 2020) implementation of the branch-and-bound algorithm to obtain the results herein.

$\boldsymbol{K}$-median clustering. The $K$-median clustering problem can be described as follows: Select $K$ of the $p$ items to serve as cluster exemplars and assign each of the items to the cluster associated with its nearest exemplar with the goal of minimizing the sum of the distances of each item to its exemplar. Accordingly, one difference between $K$-median and $K$-means clustering is that the cluster centers in the former correspond to specific items (i.e., the exemplars) whereas the cluster centers in the latter typically do not. There is also evidence that $K$-median clustering can provide better cluster recovery than $K$-means under certain conditions, such as in the cases of clustering data measured on binary variables (Brusco et al., 2017) and sliced inverse regression when outliers are present (Brusco et al., 2019). 
The distinction between exact and approximate algorithms is also relevant for $K$-median clustering. For small $p$ and $K$, one exact approach is to explicitly evaluate all combinations of exemplar subsets and select the one yielding the minimum objective function. For larger $p$ and $K$, integer linear programming is an effective method for obtaining exact solutions. An efficient heuristic procedure for the $K$-median problem was developed by Teitz and Bart (1968) and later improved by Whitaker (1983), and Hansen and Mladenović (1997). The algorithm begins with the (typically random) selection of $K$ items as cluster exemplars. Next, each item is assigned to the cluster associated with its nearest exemplar. This step is followed by an exchange process whereby each exemplar is evaluated for replacement by each of the unselected (i.e., nonexemplar) items. Any replacement that reduces the sum of the distances of the items to their exemplars is accepted. The algorithm cycles between the assignment and exchange steps until there are no changes in the set of exemplars.

Like the $K$-means heuristic, the exchange heuristic for the $K$-median problem is not guaranteed to provide a globally-optimal solution and is sensitive to the initial set of exemplars selected. Therefore, we use a multistart implementation of the exchange heuristic (see Brusco \& Köhn, 2008a, Köhn et al., 2010), whereby the algorithm is restarted 100 times using a different randomly-selected set of $K$ exemplars for each restart. This multistart approach is known to be very effective, generally producing globally-optimal solutions even for large $p$ when $K<10$ (Hansen \& Mladenović, 1997). Nevertheless, we followed the multistart exchange heuristic with a Lagrangian relaxation algorithm (see Brusco \& Köhn, 2008b; Köhn et al., 2010) in an effort to verify that the solutions obtained are indeed optimal. Specifically, MATLAB (MATLAB, 2020) implementations of the multistart exchange (mfi.m) and Lagrangian relaxation (lra.m) algorithms were used to obtain the results herein. 


\section{Motivating examples}

\section{Example \# 1: HAN depression network}

Data. Kendler et al. (2018) reported results for a network analysis of $p=19$ depression symptoms, extracted from DSM-IV and other sources, measured for a sample of Han Chinese women. ${ }^{3}$ Each respondent provided binary responses (endorsing or not endorsing) each of the 19 symptoms. The $l_{1}$-regularized logistic regression approach described by van Borkulo et al. (2014) for fitting the Ising model (Ising, 1925) was used to estimate the edge-weight matrix for the data. The edge-weight values, which were all positive and ranged from .13 to 1.71 , were published by Kendler et al. (2018) in the supplemental material associated with their article. We assembled these edge weights into a $p \times p$ adjacency matrix.

Analysis. We ran the spectral clustering algorithm spectral.m for the Han depression network using the normalized graph Laplacian of Shi and Malik (2000) in Equation 2. Table 1 reports the eigenvalues for the Laplacian as well as the eigengap measure for each value of $K$. The largest eigengap value occurs for $K=3$ and, therefore, the first three eigenvectors are also displayed in Table 1. Optimal $K$-means and $K$-median partitions were obtained using bbwcss .m and $\mathrm{mfi} . \mathrm{m}$, respectively. The partitions associated with the two optimization problems were identical. In other words, for this particular application, the results associated with $K$-median and $K$-means clustering are the same. The partition, which is displayed in Figure 1, is also the same as the partition obtained using the walktrap algorithm as reported by Kendler et al. (2018). Our MATLAB implementation of the walktrap algorithm (walktrap.m), using the maximum

\footnotetext{
${ }^{3}$ For consistency with the labeling of Kendler et al. (2018), the 19 symptoms are labeled from 2 to 20 (rather than 1 to 19$)$.
} 
similarity for each row to establish the main diagonal and a step size of $t=4$, also produced the same three-cluster partition.

[Insert Table 1 and Figure 1 About Here]

The partition in Figure 1 reveals the desirable properties that can stem from a spectral clustering of a psychological network. The blocks along the main diagonal are dense, indicating strong within-cluster cohesiveness. Moreover, it is noteworthy that the largest edge weights in the network (e.g., all edge weights exceeding 1) are between items in the same cluster. The offdiagonal blocks are comparatively sparse and the edge weights that are present tend to be smaller. Thus, although there is some overlap among the clusters, there is also a degree of separation. In addition to the desirable mathematical properties of the partition in Figure 1, Kendler et al. (2018) observed that the partition also has empirical validity. That is, the three clusters correspond to meaningful categories of depressive symptoms labeled (i) cognitive change $\{2,7,9,10,11,17\}$, (ii) neurovegetative / $\operatorname{mood}\{3,4,5,6,8,12,13,14,15\}$, and (iii) anxiety / irritability $\{16,18,20\}$.

\section{Example \#2: Obama network}

Data. Dalege et al. (2017) report the results of a network analysis of binary data pertaining to $p=10$ attitudes toward Barack Obama during the 2012 U.S. Presidential Election cycle. Like the Han depression example in the previous subsection, the network estimation process for the Obama network was accomplished using the $l_{1}$-regularized logistic regression method described by van Borkulo et al. (2014). Although some negative edges were produced by the estimation process, they were typically small in comparison to the positively-weighted edges. Dalege et al. (2017) applied the walktrap algorithm to the network after taking the absolute value of all of the edge weights. 
Analysis. We ran the spectral clustering algorithm spectral . m for the Obama network using the normalized graph Laplacian. Table 2 provides the eigenvalues for the Laplacian as well as the eigengap measure for each value of $K$. The largest eigengap value occurs for $K=4$ and, therefore, the first four eigenvectors are also displayed in Table 2. Optimal $K$-means and $K$ median partitions were obtained using bbwcss.m and $\mathrm{mfi} . \mathrm{m}$, respectively. Both algorithms yielded the same four-cluster partition: Cluster 1: \{Moral, Caring, Honest $\}$, Cluster 2: (Leadership, Intelligent, Knowledgeable\}, Cluster 3: \{Hopeful, Proud $\}$, and Cluster 4: $\{$ Angry, Afraid \}. We also clustered the network matrix using walktrap. m with a step size of $t=4$. The resulting partition, which is identical to the one reported by Dalege et al. (2017, p. 530), was slightly different from the spectral clustering partition in that 'Leadership' was positioned in Cluster 1 instead of Cluster 2. Figure 2 provides a visual representation of the partitioned network matrix.

\section{[Insert Table 2 and Figure 2]}

The dashed lines in Figure 2 are used to show that 'Leadership' is moved from Cluster 2 to Cluster 1 to obtain the walktrap.m partition from the spectral.m partition. A strong (numerical) case can be made that 'Leadership' is better positioned in Cluster 2 in accordance with the spectral clustering solution. As a general rule, we would prefer the edge weights between items in the same cluster to be large (strong within-cluster cohesion) and the edge weights between items in different clusters to be small (low between-cluster overlap). If 'Leadership' is positioned in Cluster 1 then the largest edge-weight between two attitudes not in the same cluster is 1.38 and the smallest edge weight between two attitudes in the same cluster is 0.38. Contrastingly, if 'Leadership' is positioned in Cluster 2, then the largest edge-weight 
between two attitudes not in the same cluster is 1.13 and the smallest edge weight between two attitudes in the same cluster is 0.58 .

Like the Han depression example, the eigengap heuristic resulted in the identification of a conceptually appropriate number of clusters for the Obama personality attitudes example.

Moreover, in both examples, there was no difference between the results obtained using $K$-means and $K$-median clustering of the eigenvectors of the normalized Laplacian matrix. However, a more in-depth exploration of the eigengap heuristic and the clustering algorithms is necessary, so we undertook it in a series of simulation experiments reported in the next section.

\section{Simulation experiments}

\section{General overview}

A series of four simulation experiments was undertaken to evaluate spectral clustering as a method for grouping the items associated with a psychological network. The key performance measure is the agreement between the 'true' (or known) underlying cluster structure and the spectral clustering solution. We use the adjusted Rand index (ARI: Hubert \& Arabie, 1985) as

the measure of agreement, which is recognized as the gold standard in the classification literature (Steinley, 2004; Steinley et al., 2016). The ARI is equal to one when there is perfect agreement between two partitions and is near zero when there is only chance agreement. For simulation studies, Steinley (2004) provided guideline thresholds of 0.90, 0.80 and 0.65 for excellent, good, and fair agreement, respectively.

For each of the four experiments, the networks consisted of $p=24$ items. Although some studies have more items and many have fewer, $p=24$ is a reasonable representation of network size in the network psychometrics literature. Moreover, this network size enables us to use the exact methods for $K$-means and $K$-median clustering, such that our results cannot be muddied by 
potential suboptimality for some problems. Using a larger network size might preclude the use of exact methods because of the computational requirement necessary to solve a large number of problems. Three problem-design features were used in each of the four experiments: (i) number of clusters, (ii) within-cluster cohesion, and (iii) overlap between clusters.

The number of clusters was tested at five levels: $K=2, K=3, K=4, K=5$, and $K=6$. Two clusters are the standard minimum in most simulation experiments and more than six clusters is likely too many for $p=24$ items. Within-cluster cohesion was measured by the probability of an edge connecting two items in the same cluster. The higher the probability, the greater the level of cohesion. The three levels for the within-cluster cohesion design feature were 0.9 (high), 0.8 (medium), and 0.7 (low). Edge weights for the selected within-cluster edges were randomly selected from a uniform distribution on the interval $[0.4,1.0]$. Cluster overlap was measured by the probability of an edge connecting two items in different clusters. The higher the probability, the greater the level of overlap. The three levels for the cluster overlap design feature were 0.1 (low), 0.2 (medium), and 0.3 (high). The experimental design consisted of $5 \times 3 \times 3=45$ cells and we used 10 replications per cell, resulting in a total of 450 test problems for each of the simulation experiments.

Six different methods were applied to each of the 450 test problems: (1) the walktrap algorithm $(t=4)$ using hierarchical clustering, (2) the walktrap algorithm $(t=4)$ using branchand-bound programming, (3) spectral clustering using the unnormalized Laplacian and $K$-means clustering, (4) spectral clustering using the unnormalized Laplacian and $K$-median clustering, (5) spectral clustering using the normalized Laplacian and $K$-means clustering, and (6) spectral clustering using the normalized Laplacian and $K$-median clustering, The four experiments were 
conducted in MATLAB and all of the m-files for their implementation are available from the authors.

\section{Simulation 1: $K$ known, equally-sized clusters}

The first simulation experiment was conducted using the common assumption that the appropriate (true) number of clusters is known. In addition, the number of items in each cluster was equal. ${ }^{4}$ For each of the 450 test problems, results were obtained for both the unnormalized and normalized Laplacian matrices, and using both $K$-means and $K$-median clustering for the partitioning of the items in Step 3. The results for Simulation 1 are summarized in Table 3. The top panel of Table 3 provides the average ARI for each type of Laplacian matrix and each clustering method. The overall (i.e., across all 450 test problems) averages are provided, along with the average for each level of each design feature. The bottom panel of Table 3 provides the percentage of test problems for which perfect (i.e., ARI =1) was achieved.

\section{[Insert Table 3 About Here]}

The results in Table 3 indicate that, for spectral clustering, the normalized Laplacian matrix resulted in much better cluster recovery than the unnormalized Laplacian matrix. However, for both Laplacian matrices, there was very little difference in performance between $K$-means and $K$ median clustering. When using the normalized Laplacian matrix, the average ARI values for the $K$-means and $K$-median clustering methods were .9404 and .9415 , respectively. Contrastingly, when using unnormalized Laplacian matrix, the corresponding averages for these methods were .8691 and .8695 , respectively. Moreover, for both clustering methods, the average ARI for the normalized Laplacian matrix was at least as large (and often appreciably larger) than the average ARI for the unnormalized Laplacian matrix for each level of each experimental design feature.

\footnotetext{
${ }^{4}$ The number of items in each cluster was exactly equal to $24 / K$ for $K=2, K=3, K=4$, and $K=6$ test problems, For the $K=5$ condition, the first four clusters each had 5 items and the fifth cluster had four items.
} 
Similar findings were observed for the percentage of problems for which perfect recovery (i.e., $\mathrm{ARI}=1)$ was realized. When using the normalized Laplacian matrix, the $K$-means and $K$-median clustering methods yielded perfect recovery for $74.4 \%$ and $74.7 \%$ of the test problems, respectively. The comparative figures for the unnormalized Laplacian matrix were $62.0 \%$ and $62.2 \%$, respectively.

The results in Table 3 suggest that the recovery performance of the walktrap algorithm can be improved slightly by replacing Ward's method-type hierarchical clustering with exact $K$ means clustering. The average ARI values for the walktrap algorithm when using hierarchical clustering and exact $K$-means partitioning were .9189 and .9264 , respectively. Likewise, these two implementations of the walktrap algorithm yielded perfect recovery for $68.2 \%$ and $71.3 \%$ of the test problems, respectively. Accordingly, both implementations of the walktrap algorithm substantially outperformed spectral clustering using the unnormalized Laplacian matrix. However, both implementations of the walktrap algorithm were outperformed by spectral clustering using the normalized Laplacian matrix.

The performances of all methods were profoundly influenced by the levels of the design features. For all methods, cluster recovery (as measured by both average ARI and the percentage of problems for which perfect recovery was realized) generally decreased as $K$ increased over the range from 2 to 6 . Likewise, recovery decreased as within-cluster cohesion decreased across the levels $0.9,0.8$, and 0.7 and recovery decreased as cluster overlap increased across the levels 0.1 , 0.2 , and 0.3 .

\section{Simulation 2: $K$ known, unequally-sized clusters}

The second simulation experiment was also conducted under the assumption that the appropriate (true) number of clusters is known. However, in this experiment, the number of items in each cluster was unequal. More specifically, one of the clusters was very small, 
consisting of only two items, and all other clusters were of equal (or as equal as possible) size. This type of occurrence is not uncommon. For example, in the Han depression example described in the previous section, there was one small cluster consisting of only three items. Other than distribution of items across clusters, the experimental design of Simulation 2 was identical to that of Simulation 1. The results of Simulation 2 are summarized in Table 4 .

\section{[Insert Table 4 About Here]}

Table 4 reveals that, once again, the normalized Laplacian matrix resulted in better cluster recovery than the unnormalized Laplacian matrix. When using the normalized Laplacian matrix, the average ARI values for the $K$-means and $K$-median clustering methods were .9489 and .9495 , respectively. Contrastingly, when using unnormalized Laplacian matrix, the corresponding averages for these methods were .8947 and .8765 , respectively. Likewise, when using the normalized Laplacian matrix, the $K$-means and $K$-median clustering methods yielded perfect recovery for $74.0 \%$ and $73.6 \%$ of the test problems, respectively. The comparative figures for the unnormalized Laplacian matrix were $62.4 \%$ and $53.1 \%$, respectively.

The two versions of the walktrap algorithm also yielded excellent recovery in Simulation 2, with a slight improvement realized from using exact $K$-means instead of hierarchical clustering. The average ARI values for the walktrap algorithm when using hierarchical clustering and exact $K$-means partitioning were .9340 and .9369 , respectively. Likewise, these two implementations of the walktrap algorithm yielded perfect recovery for $71.3 \%$ and $71.8 \%$ of the test problems, respectively. Once again, both versions of the walktrap algorithm substantially outperformed spectral clustering using the unnormalized Laplacian matrix. Although spectral clustering using the normalized Laplacian matrix yielded better recovery than the walktrap algorithm, both 
overall and for most (but not all) levels of the design features, the disparity in performance was less than it was in Simulation 1.

Like Simulation 1, the results for Simulation 2 exhibited a propensity for reduction in cluster recovery as $K$ increased, as within-cluster cohesion decreased, and as cluster overlap increased. Spectral clustering using the normalized Laplacian matrix held its largest average ARI advantages over its competitors at settings of $K=6$ clusters and cluster overlap probability of 0.3 .

\section{Simulation 3: K unknown, equally-sized clusters}

The third simulation experiment was identical to Simulation 1, with the exception that the appropriate (true) number of clusters was unknown. Accordingly, for each test problem in Simulation 3, the number of clusters for the walktrap algorithm was selected based on the ratio rule and the number of clusters for the spectral clustering methods was selected based on the eigengap heuristic. The cluster recovery results of Simulation 3 are summarized in Table 5. The results pertaining to the correct identification of the number of clusters is provided in Table 6 . For both the ratio rule (walktrap algorithm) and the eigengap heuristic (spectral clustering for both the unnormalized and normalized Laplacian matrices), Table 6 displays the percentage of test problems for which the number of clusters in the network $\left(K^{*}\right)$ was underestimated $\left(K<K^{*}\right)$, correctly estimated $\left(K=K^{*}\right)$, and overestimated $\left(K>K^{*}\right)$.

\section{[Insert Tables 5 and 6 About Here]}

The results in Table 6 indicate that, when using the normalized Laplacian matrix, the eigengap heuristic obtained the correct number of clusters for $85.3 \%$ of the test problems. Too many clusters were selected for $5.3 \%$ of the problems and too few were chosen for $9.3 \%$ of the problems. Contrastingly, when using the unnormalized Laplacian matrix, the eigengap heuristic obtained the correct number of clusters for only $58.2 \%$ of the test problems. Too many clusters 
were selected for $28.9 \%$ of the problems and too few were chosen for $12.9 \%$ of the problems. The ratio rule selected the correct number of clusters for the walktrap algorithm for $74.4 \%$ of the test problems, while too many and too few clusters were selected for $7.3 \%$ and $18.2 \%$ of the problems, respectively.

In light of the fact that the eigengap heuristic selected the correct number of clusters for more than $85 \%$ of the test problems, cluster recovery for the spectral clustering using the normalized Laplacian matrix remained excellent. Table 5 reveals that, when using the normalized Laplacian matrix, the average ARI values for the $K$-means and $K$-median clustering methods were .9133 and .9128 (compared to .9404 and .9415 in Simulation 1). The impact on recovery was more severe for the walktrap algorithm, where the average ARI values for the hierarchical and exact $K$-means clustering methods were .8635 and .8679 (compared to .9189 and .9264 in Simulation 1). Interestingly, although worse than the other methods with respect to the identification of the correct number of clusters, the decrease in average ARI performance for spectral clustering using the unnormalized Laplacian matrix was less severe, with corresponding averages for $K$-means and $K$-median methods of .8458 and .8471 (compared to .8691 and .8695 in Simulation 1), respectively.

Like Simulation 1, the $K$-means and $K$-median methods were comparable with respect to their recovery performance with respect to both average ARI and the percentage of problems for which perfect recovery was achieved. For the walktrap algorithm, the exact $K$-means approach yielded slightly better recovery than hierarchical clustering. For all methods, cluster recovery generally decreased as $K$ increased over the range from 2 to 6 . Likewise, recovery decreased as within-cluster cohesion decreased across the levels $0.9,0.8$, and 0.7 and recovery decreased as cluster overlap increased across the levels $0.1,0.2$, and 0.3 . 


\section{Simulation 4: $K$ unknown, unequally-sized clusters}

The fourth simulation experiment was identical to Simulation 2, with the exception that the appropriate (true) number of clusters was assumed to be unknown. Accordingly, for each test problem in Simulation 4, the number of clusters for the walktrap algorithm was selected based on the ratio rule and the number of clusters for the spectral clustering methods was selected based on the eigengap heuristic. The cluster recovery results of Simulation 3 are summarized in Table 7. The results pertaining to the correct identification of the number of clusters is provided in Table 8 .

\section{[Insert Tables 7 and 8 About Here]}

The unequal cluster sizes and need to select $K$ resulted in Simulation 4 posing the most challenging test conditions. The results in Table 8 reveal that the percentage of test problems for which the eigengap correctly selected the true number of clusters was $54.4 \%$ for the normalized Laplacian matrix and $34.4 \%$ for the unnormalized Laplacian matrix. However, there was a tremendous disparity with respect to the direction of mis-selection of the number of clusters. For the normalized Laplacian matrix, the eigengap heuristic selected too many clusters for $2.9 \%$ of the problems and too few for $42.7 \%$ of the problems. For the unnormalized Laplacian matrix, the eigengap heuristic selected too many clusters for $49.3 \%$ of the problems and too few for $16.2 \%$ of the problems. Using the walktrap algorithm with the ratio rule resulted in the correct selection of the number of clusters for $56.4 \%$ of the test problems, while too many and too few clusters were selected for $10.2 \%$ and $33.3 \%$ of the problems, respectively.

Once again, because selecting too few clusters tends to have more serious ramifications for ARI than selecting too many, the average ARI performances for spectral clustering using the normalized Laplacian matrix and the walktrap algorithm were more severely diminished relative to the results for the unnormalized Laplacian matrix. Table 7 reveals that, when using the 
normalized Laplacian matrix, the average ARI values for the $K$-means and $K$-median clustering methods were .8745 and .8729 (compared to .9489 and .9495 in Simulation 2). The impact on recovery was also severe for the walktrap algorithm, where the average ARI values for the hierarchical and exact $K$-means clustering methods were .8708 and .8719 (compared to .9340 and .9369 in Simulation 2). The decrease in average ARI performance for spectral clustering using the unnormalized Laplacian matrix was less severe, with corresponding averages for $K$ means and $K$-median methods of .8636 and .8551 (compared to .8947 and .8765 in Simulation 2), respectively.

\section{Discussion}

The simulation experiments in the previous section have provided several insights regarding the use of spectral clustering and the walktrap algorithms for psychometric networks. The key decisions/questions a researcher faces when using these methods are: (1) How should the network adjacency matrix be obtained? (2) How should the weights for loops in the adjacency matrix be determined? (3) Are there benefits to replacing hierarchical clustering in the walktrap algorithm with an exact method? (4) Which type of Laplacian matrix should be used for spectral clustering? (5) Should $K$-means or $K$-median clustering be used in Step 3 of spectral clustering?

(6) How should the number of clusters be selected for the walktrap algorithm and spectral clustering? (7) How do the walktrap algorithm and spectral clustering compare in terms of cluster recovery?

The answer to the first question has been the focus of significant attention in the network psychometrics literature. Adjacency matrices are produced using methods such as regularized Gaussian models (Friedman et al., 2008), regularized logistic regression models (van Borkulo et al., 2014), and nonregularized partial correlation methods (Williams \& Rast, 2020; Williams et 
al., 2019). These methods often produce adjacency matrices with reasonable levels of density (e.g., 30-70\% of possible edges are estimated) and, accordingly, spectral clustering can be applied directly to such matrices. Other approaches, such as relative importance networks (Grömping, 2006) typically estimate complete graphs, in which case it will be likely be necessary to apply some type of thresholding procedure to produce a graph that is neither too dense or too sparse (Chen et al., 2017).

Regarding the second question, most psychometric networks published in the literature do not display or report loops for the adjacency matrices. Nevertheless, they are required for the walktrap algorithm. Using the maximum values for each row of the adjacency matrix to establish the main diagonal enabled us to match the walktrap algorithm results for the Han depression (Kendler et al., 2018) and Obama (Dalege et al., 2017) networks. The maximum value has a conceptual appeal because it means that the self-tie (i.e., the loop) is exactly as strong as the strongest tie of that vertex to any other vertex. We also tried using the row averages and sums instead of the row maximums but found them to perform poorly.

Regarding the third question, the simulations showed a very modest improvement in recovery associated with using an exact $K$-means clustering method instead of the hierarchical approach based on Ward's method as proposed by Pons and Latapy (2006). This modest improvement, in conjunction with the scalability advantages of the hierarchical algorithm, lead us to conclude that continued use of the hierarchical algorithm in network psychometric applications is reasonable.

Regarding the fourth question, our simulation results indicate that the normalized Laplacian matrix (Shi \& Malik, 2000) leads to better cluster recovery than the unnormalized Laplacian matrix (Mohar, 1997). The average ARI's for the normalized Laplacian were appreciably better 
in Simulations 1 and 2 where the true number of clusters was specified. The normalized Laplacian also generally provided better results in Simulation 3 under the conditions of equal cluster size and the use of the eigengap heuristic to choose $K$. Although the unnormalized Laplacian led to only slightly inferior average ARI results in Simulation 4, this was attributable to over- and under-estimation properties of the eigengap heuristic rather than superiority of the unnormalized Laplacian matrix.

Regarding the fifth question, the performances of the $K$-means and $K$-median methods were comparable across most of the simulation settings. There was certainly no compelling evidence to warrant a shift away from the more familiar and more widely accessible $K$-means clustering method to the less well-known $K$-median method. However, if a researcher desires to have an item from each cluster to serve as the 'exemplar' (center) for their cluster, then this is directly provided from the $K$-median clustering method.

Regarding the sixth question, in many network psychometrics applications, theory may guide decisions surrounding an appropriate number of clusters or communities. Under such circumstances, the values of $K$ might be prespecified or perhaps tested within a very narrow range. When there is less certainty regarding the value of $K$, the eigengap rule can be used to choose the number of clusters for spectral clustering, whereas the ratio rule can be used for such purposes when using the walktrap algorithm. In Simulation 3, the eigengap rule worked exceptionally well when the cluster sizes were equal and the normalized Laplacian was used, yielding the correct number of clusters for more than $85 \%$ of the data sets. The ratio rule yielded the correct number of clusters for nearly $75 \%$ of the data sets. These percentages dropped significantly in Simulation 4 when the clusters were not equally sized. It was encouraging, however, that despite the decrease in the percentage of problems for which perfect recovery was 
realized, the recovery of cluster structure was not overly compromised. That is, average ARI values remained reasonably strong despite the common mis-selection of $K$. Nevertheless, future research should explore alternative approaches for choosing $K$. The evaluation of other measures of differences (or ratios) between changes in the eigenvalues is one possible option (Brusco et al., 2017; Dimitriadou et al., 2002). More computationally-demanding procedures such as the gap statistic (Tibshirani et al., 2001) have also been proposed (Chen et al., 2017).

Regarding the seventh question, the simulation results provide strong fortification for the use of the walktrap algorithm in network psychometrics. Although the average ARI and percentage of perfectly-recovered cluster structures for the walktrap versions in Simulations 1 and 2 were slightly lower than the corresponding figures for spectral clustering using the normalized Laplacian matrix, the recovery performances were still very compelling. Moreover, the walktrap algorithm substantially outperformed spectral clustering using the unnormalized Laplacian matrix in both Simulations 1 and 2. Nevertheless, across all four simulation experiments, spectral clustering using the normalized Laplacian matrix was the top performing approach. This fact, in conjunction with the interesting partition we obtained for the Obama network, suggests that spectral clustering deserves due consideration for network psychometrics.

\section{Conclusions}

\section{Summary}

Spectral clustering is a well-established for method for obtaining a partition of the vertices of network matrices. As described by von Luxburg (2017, pp. 404-405), the normalized Laplacian proposed by Shi and Malik (2000) is closely related to the transition matrix for a random walk on a similarity graph (Lovász, 1993). Accordingly, spectral clustering is related to the walktrap algorithm of Pons and Latapy (2006, p. 198), who note that their method is based on the same foundation as spectral clustering. The walktrap algorithm has been widely used in network 
psychometrics; however, the motivation for its original development focused on computational feasibility relative to spectral clustering via the circumvention of the computation of eigenvectors for large sparse matrices. In light of the fact that psychological network matrices are comparatively small and eigenvector computation for them is not computationally demanding, it seems reasonable to give consideration to the use of spectral clustering in this application domain and compare its performance to the walktrap algorithm.

We began our analyses with two motivating examples from the network psychometrics literature. In the first example, we found that, in this particular application, spectral clustering led to the same walktrap algorithm partition reported in the original article (Kendler et al., 2018). In the second example, spectral clustering led to a slightly different partition than the walktrap algorithm that, arguably, had greater appeal (at least from a numerical standpoint).

Next, we conducted a series of simulation experiments to evaluate spectral clustering and compare its performance to the walktrap algorithm. We considered several implementation decisions regarding spectral clustering, such as the choice of the Laplacian matrix, the selection of the number of clusters, and the algorithm used to cluster the eigenvectors. Each simulation consisted of 450 test problems where we varied three design features: the number of clusters in the network, the within-cluster cohesion of the network, and the between-cluster overlap. The results indicated that (i) cluster recovery was sensitive to each of the design features, (ii) cluster recovery was sensitive to the relative sizes of the clusters, (iii) the normalized Laplacian matrix was generally superior to its unnormalized counterpart, (iv) the eigengap heuristic for choosing the number of clusters performed extremely well for equally-sized clusters but was less effective when clusters were not equally-sized, (v) the $K$-means and $K$-median methods performed comparably, and (vi) the walktrap substantially outperformed spectral clustering using the 
unnormalized Laplacian matrix, but was slightly outperformed by spectral clustering using the normalized Laplacian matrix.

\section{Limitations and extensions}

All clustering methods have limitations. In the case of spectral clustering, it has been noted that the method can fail when used for large networks that are very sparse (Fortunato \& Hric, 2016). However, a major motivation of our paper is that psychological networks are generally neither large nor very sparse. Accordingly, we believe that this particular limitation will seldom apply to the 10-to-50-node networks commonly encountered in network psychometrics literature.

Our simulation study has revealed one potential problem area of spectral clustering, namely the selection of the number of clusters. The eigengap heuristic works well for some data conditions but not others. This finding is consistent with studies pertaining to the selection of the number of clusters for other clustering methods (Brusco et al., 2017; Dimitriadou et al. 2002;

Köhn et al., 2010; Milligan \& Cooper, 1985; Steinley \& Brusco, 2011). A comparative analysis of alternative rules for choosing the number of clusters for a spectral clustering is a worthwhile avenue for future research.

Another limitation of spectral clustering, as well as similar approaches such as the walktrap algorithm, is that Step 3 of the algorithm produces a partition of the items. Fried (2016) has noted that there is more likely to be overlap between clusters, with some items having multiple cluster memberships. The clique percolation method (Palla et al., 2005) is one approach for accomplishing this goal. However, variants of spectral clustering that permit cluster overlap have been developed and may also be of practical value to address this problem (Kaufmann et al., 2018). Overlapping clustering has a rich history in the psychological literature (Shepard \& 
Arabie, 1979) and we believe that further exploration of overlapping clustering methods in psychological network research might be of considerable value. 


\section{References}

Blanken, T. F., Deserno, M. K., Dalege, J., Borsboom, D., Blanken, P., Kerkhof, G. A., \& Cramer, A. O. J. (2018). The role of stabilizing and communicating symptoms given overlapping communities in psychopathology networks. Scientific Reports, 8, 59. doi:10.1038/s41598-018-24224-2

Borg, I., Groenen, P. J. F., \& Mair, P. (2018). Applied multidimensional scaling and unfolding, 2nd ed. New York, NY: Springer Science \& Business Media. doi: 10.1007/978-3-31973471-2

Briganti, G., Kempenaers, C., Braun, S., Fried, E. I., Linkowski, P. (2018). Network analysis of empathy items from the interpersonal reactivity index in 1973 young adults. Psychiatry Research, 265, 87-92. doi: 10.1016/j.psychres.2018.03.082

Brusco, M. J. (2006). A repetitive branch-and-bound algorithm for minimum within-cluster sums of squares partitioning. Psychometrika, 71 (2), 347-363. https://doi.org/10.1007/s11336004-1218-1

Brusco, M. J., \& Kohn, H.-F. (2008a). Comment on 'Clustering by passing messages between data points'. Science, 319, 726c. https://doi.org/10.1126/science.1150938

Brusco, M. J., \& Kohn, H.-F. (2008b). Optimal partitioning of a data set based on the $p$-median model. Psychometrika, 73 (1), 89-105. https://doi.org/10.1007/s11336-007-9021-4

Brusco, M. J., Shireman, E., \& Steinley, D. (2017). A comparison of latent class, $K$-means, and $K$-median methods for clustering dichotomous data. Psychological Methods, 22, 563580. https://doi.org/10.1037/met0000095.

Brusco, M. J., \& Stahl, S. (2005). Branch-and-bound applications in combinatorial data analysis. New York: Springer.

Brusco, M. J., Steinley, D., \& Stevens, J. (2019). K-medoids inverse regression. Communications in Statistics - Theory and Methods, 48 (20), 4999-5011. https://doi.org/10.1080/03610926.2018.1504076

Brusco, M. J., Steinley, D., \& Watts, A. L. (in press). Disentangling relationships in symptom networks using matrix permutation methods. Psychometrika.

Ceulemans, E., \& Van Mechelen, I. (2005). Hierarchical classes models for three-way threemode binary data: Interrelations and model selection. Psychometrika, 70, 461-480. http://dx.doi.org/10.1007/s11336-003-1067-3

Chen, Y., Li, X., Liu, J., Xu, G., \& Ying, Z. (2017). Exploratory item classification via spectral graph clustering. Applied Psychological Measurement, 41 (8), 579-599. https://doi.org/10.1177/ 014662/617692977.

Choi, K. W., Batchelder, A. W., Ehlinger, P. P., Safren, S. A., \& O’Cleirigh, C. (2017). Applying network analysis to psychological comorbidity and health behavior: Depression, PTSD, and sexual risk in sexual minority men with trauma histories. Journal of Consulting and Clinical Psychology, 85, 1158-1170. doi: 10.1037/ccp0000241 
Christensen, A. P. (2018). NetworkToolbox: Methods and measures for brain, cognitive, and psychometric network analysis in R. The R Journal, 10 (2), 422-439. Retrieved 2/17/2021 from: https://journal.r-project.org/archive/2018/RJ-2018-065/RJ-2018-065.pdf

Cramer, A. O., Waldorp, L. J., van der Maas, H. L., \& Borsboom, D. (2010a). Comorbidity: A network perspective. Behavioral and Brain Sciences, 33(2-3), 137-150. doi: 10.1017/S0140525X09991567.

Cramer, A. O., Waldorp, L. J., van der Maas, H. L., \& Borsboom, D. (2010b). Complex realities require complex theories: Refining and extending the network approach to mental disorders. Behavioral and Brain Sciences, 33(2-3), 178-193. doi:10.1017/S0140525X10000920

Csardi, G., \& Nepusz, T. (2006). The igraph software package for complex network research. InterJournal Complex Systems, 1695, 1-9.

Dalege, J., Borsboom, D., van Harreveld, F., \& van der Maas, H. J. L. (2017). Network analysis on attitudes: a brief tutorial. Social Psychological and Personality Science, 8 (5), 528537. doi: 10.1177/1948550617709827

Dimitriadou, E., Dolničar, S., \& Weingessel, A. (2002). An examination of indices for determining the number of clusters in binary data sets. Psychometrika, 67, 137-159. http://dx.doi.org/10.1007/BF02294713

Epskamp, S., \& Fried, E. I. (2018). A tutorial on regularized partial correlation networks. Psychological Methods, 23(4), 617-634. doi:10.1037/met0000167

Forgy, E. W. (1965). Cluster analyses of multivariate data: Efficiency versus interpretability of classifications. Biometric Society Meeting, Riverside, CA, 1965. Abstract in Biometrics, 21,768 .

Fortunato, S., \& Hric, D. (2016). Community detection in networks: a user's guide. Physics Reports, 659, 1-44. https://doi.org/10.1016/j.physrep.2016.09.002

Fried, E. I. (2016). R tutorial: how to identify communities of items in networks. Retrieved from http://psych-networks.com/r-tutorial-identify-communities-items-networks/

Friedman, J. H., Hastie, T., \& Tibshirani, R. (2008). Sparse inverse covariance estimation with the graphical lasso. Biostatistics, 9(3), 432-441. doi: 10.1093/biostatistics/kxm045

Friedman, J. H., Hastie, T., \& Tibshirani, R. (2014). glasso: Graphical lasso-estimation of Gaussian graphical models (R package version 1.8). Retrieved from https://CRAN.Rproject.org/package_glasso

Fruchterman, T. M. J., \& Reingold, E. M. (1991). Graph drawing by force-directed placement. Software, Practice \& Experience, 21, 1129-1164. http://dx.doi.org/10.1002/spe.4380211102

Golino, H., \& Demetriou, A. (2017). Estimating the dimensionality of intelligence like data using exploratory graph analysis. Intelligence, 62, 54-70. https://doi.org/10.1016/j.intell.2017.02.007

Golino, H. F., \& Epskamp, S. (2017). Exploratory graph analysis: A new approach for estimating the number of dimensions in psychological research. PloS One, 12(6), :e0174035, 2017. URL https://doi.org/10.1371/journal.pone.0174035. 
Grömping, U. (2006). Relative importance for linear regression in R: The package relaimpo. Journal of Statistical Software, 17, 1-27. doi:10.18637/jss.v017.i01

Hakimi, S. L. (1964). Optimum locations of switching centers and the absolute centers and medians of a graph. Operations Research, 12, 450-459. https://doi.org/10.1287/opre.12.3.450.

Hakimi, S. L. (1965). Optimum distribution of switching centers in a communication network and some related graph theory problems. Operations Research, 13, 462-475. https://doi.org/10. 1287/opre.13.3.462.

Hand, D. J. (1981). Branch and bound in statistical data analysis, The Statistician, 30, 1-13. https://doi.org/10.2307/2987699

Hansen, P., \& Mladenović, N. (1997). Variable neighborhood search for the p-median. Location Science, 5, 207-226. https://doi.org/10.1016/S0966-8349(98)00030-8

Hevey, D. (2018). Network analysis: a brief overview and tutorial. Health Psychology and Behavioral Medicine, 6(1), 301-328. https://doi.org/10.1080/21642850.2018.1521283

Hoff, P., Raftery, A. E., \& Handcock, M. S. (2002). Latent space approaches to social network analysis. Journal of the American Statistical Association, 97, 1090-1098. doi: 10.1198/016214502388618906

Hoffman, M., Steinley, D., Gates, K. M., Prinstein, M. J., \& Brusco, M. J. (2018). Detecting clusters/communities in social networks. Multivariate Behavioral Research, 53 (1), 57 73. doi:10.1080/00273171.2017.1391682

Hubert, L., \& Arabie, P. (1985). Comparing partitions. Journal of Classification, 2, 193-218. https://doi.org/10.1007/BF01908075

Ising, E. (1925). Beitrag zur theorie des ferromagnetismus. Zeitschrift für Physik, 31(1), 253258. doi:10.1007/BF02980577

Jones, P. J., Mair, P., \& McNally, R. J. (2018a). Visualizing psychological networks: A tutorial in R. Frontiers in Psychology, https://doi.org/10.3389/fpsyg.2018.01742.

Jones, P. J., Mair, P., Riemann, B. C., Mungo, B. L., \& McNally, R. J. (2018b). A network perspective on comorbid depression in adolescents with obsessive-compulsive disorder. Journal of Anxiety Disorders, 53, 1-8. http://dx.doi.org/10.1016/j.janxdis.2017.09.008

Kendler, K. S., Aggen, S. H., Flint, J., Borsboom, D., \& Fried, E. I. (2018). The Centrality of DSM and non-DSM Depressive Symptoms in Han Chinese Women with Major Depression. Journal of Affective Disorders, 227, 739-744. doi: 10.1016/j.jad.2017.11.032

Kaufmann, E., Bonald, T., \&, Lelarge, M. (2018). A spectral algorithm with additive clustering for the recovery of overlapping communities in networks. Theoretical Computer Science, 742, 3-26. https://doi.org/10.1016/j.tcs.2017.12.028

Kohn, H.-F., Steinley, D., \& Brusco, M. J. (2010). The $p$-median model as a tool for clustering psychological data. Psychological Methods, 15, 87-95. https://doi.org/10.1037/a0018535.

Lancichinetti, A., \& Fortunato, S. (2011). Limits of modularity maximization in community detection. Physical Review E, 84(6), 066122.doi: 10.1103/PhysRevE.84.066122 
Lovász, L. (1993). Random walks on graphs: a survey. In: Combinatorics, Paul Erdös is Eighty: Bolyai Society of Mathematical Studies, Vol. 2 (Keszthely), (pp. 353-397), János Boylai Mathematical Society: Budapest, Hungary.

MacQueen, J. B. (1967). Some methods for classification and analysis of multivariate observations. In L. M. Le Cam \& J. Neyman (Eds.), Proceedings of the fifth Berkeley symposium on mathematical statistics and probability (Vol. 1, pp. 281-297). Berkeley, CA: University of California Press.

Marsman, M., Borsboom, D., Kruis, J., Epskamp, S., van Bork, R., Waldorp, L. J., van der Maas, H. L. J., \& Maris, G. (2018). An introduction to network psychometrics: Relating Ising models to item response theory models. Multivariate Behavioral Research, 53(1), 15-35. doi: 10.1080/00273171.2017.1379379

Marsman, M., Maris,G., Bechger, T., \& Glas, C. (2015). Bayesian inference for low-rank Ising networks. Scientific Reports, 5(9050), 1-7. doi: 10.1038/srep09050

MATLAB. (2020). version 9.8.0 (R2020a). Natick, Massachusetts: The MathWorks Inc.

McElroy, E., \& Patalay, P. (2019). In search of disorders: internalizing symptom networks in a large clinical sample. Journal of Child Psychology and Psychiatry, 60 (8), 897-906. doi:10.1111/jcpp.13044

McNally, R. J. (2016). Can network analysis transform psychopathology? Behaviour Research and Therapy, 86, 95-104. doi: 10.1016/j.brat.2016.06.006

Milligan, G. W., \& Cooper, M. C. (1985). An examination of procedures for determining the number of clusters in a data set. Psychometrika, 50, 159-179. https://doi.org/10.1007/BF02294245

Mohar, B. (1991). The Laplacian spectrum of graphs. In Y. Alavi et al. (Eds.), Graph theory, combinatorics, and applications (Vol. 2, pp. 871-898), New York: Wiley.

Mohar, B. (1997). Some applications of Laplace eigenvalues of graphs. In G. Hahn \& G. Sabidussi (Eds.), Graph symmetry: Algebraic methods and applications (Vol. 497, pp. 225-275), Dordrecht, The Netherlands: Kluwer Academic Publishers.

Newman, M. E. (2004). Fast algorithm for detecting community structure in networks. Physical Review E, 69(6), 066133.doi: 10.1103/PhysRevE.69.066133

Newman M. E., \& Girvan, M. (2004). Finding and evaluating community structure in networks. Physical Review E, 69(2), 026113.doi: 10.1103/PhysRevE.69.026113

Palla, G., Derényi, I., Farkas, I. \& Vicsek, T. (2005). Uncovering the overlapping community structure of complex networks in nature and society. Nature, 435, 814-818. https://doi.org/10.1038/nature03607

Pons, P., \& Latapy, M. (2006). Computing communities in large networks using random walks. Journal of Graph Algorithms and Applications, 10 (2), 191-218. http://jgaa.info/accepted/2006/PonsLatapy2006.10.2.pdf

Reichardt, J., \& Bornholdt, S. (2006). Statistical mechanics of community detection. Physical Review E: Statistics and Nonlinear Soft Matter Physics, 74, 016110-1-016110-14 
Scutari, M. (2010). Learning Bayesian networks with the bnlearn R package. Journal of Statistical Software, 35, 1-22. Retrieved from http://www.jstatsoft.org/v35/i03/

Shepard, R. N., \& Arabie, P. (1979). Additive clustering: Representation of similarities as combinations of discrete overlapping properties. Psychological Review, 86(2), 87-123. doi.org/10.1037/0033-295X.86.2.87

Shi, J., \& Malik, J. (2000). Normalized cuts and image segmentation. IEEE Transactions on Pattern Analysis and Machine Intelligence, 22, 888-905. https://doi.org/10.1109/34.868688.

Steinhaus, H. (1956). Sur la division des corps matériels en parties [On the separation of objects into groups]. Bulletin de l'Académie Polonaise des Sciences, Classe III, IV(12), 801-804.

Steinley, D. (2003). Local optima in $K$-means clustering: What you don't know may hurt you. Psychological Methods, 8(3), 294-304. https://doi.org/10.1037/1082-989X.8.3.294

Steinley, D. (2004). Properties of the Hubert-Arabie adjusted Rand index. Psychological Methods, 9, 386-396. doi: 10.1037/1082-989X.9.3.386

Steinley, D. (2006). K-means clustering: A half-century synthesis. British Journal of Mathematical and Statistical Psychology, 59(1), 1-34. https://doi.org/10.1348/000711005X48266

Steinley, D., \& Brusco, M. J. (2011). Choosing the number of clusters in $K$-means clustering. Psychological Methods, 16, 271-285. doi:10.1037/a0023346

Steinley, D., Brusco, M. J., \& Hubert, L. (2016). The variance of the adjusted Rand index. Psychological Methods, 21 (2), 261-272. doi: 10.1037/met0000049

Teitz, M. B., \& Bart, P. (1968). Heuristic methods for estimating the generalized vertex median of a weighted graph. Operations Research, 16, 955-961. https://doi.org/10.1287/opre.16.5.955

Thorndike, R. L. (1953). Who belongs in the family? Psychometrika, 18, 267-276. https://doi.org/10.1007/BF02289263

Tibshirani, R., Walther, G., \& Hastie, T. (2001). Estimating the number of clusters in a data set via the gap statistic. Journal of the Royal Statistical Society, Series B: Statistical Methodology, 63, 411-423. doi:10.1111/1467-9868.00293

van Borkulo, C. D., Borsboom, D., Epskamp, S., Blanken, T. F., Boschloo, L., Schoevers, R. A., \& Waldorp, L. J. (2014). A new method for constructing networks from binary data. Scientific Reports, 4(5918), 1-10. doi:10.1038/srep05918.

von Luxburg, U. (2007). A tutorial on spectral clustering. Statistics and Computing, 17 (4), 395416. https://doi.org/10.1007/s11222-007-9033-z

Ward, J. H. (1963). Hierarchical grouping to optimize an objective function. Journal of the American Statistical Association, 58 (301), 236-244. https://doi.org/10.2307/2282967

Whitaker, R. (1983). A fast algorithm for the greedy interchange of large-scale clustering and median location problems. INFOR, 21, 95-108.

https://doi.org/10.1080/03155986.1983.11731889 
Wilderjans, T. F., Ceulemans, E., \& Meers, K. (2013). CHull: A generic convex-hull-based model selection method. Behavior Research Methods, 45, 1-15. http://dx.doi.org/10.3758/s13428-012-0238-5

Williams, D. R., \& Rast, P. (2020). Back to the basics: Rethinking partial correlation network methodology. British Journal of Mathematical and Statistical Psychology, 73, 187-212. doi:10.1111/bmsp.1217

Williams, D. R., Rhemtulla, M., Wysocki, A. C., \& Rast, P. (2019). On nonregularized estimation of psychological networks. Multivariate Behavioral Research, 54 (5), 719 750. doi: 10.1080/00273171.2019.1575716 
Table 1. The eigenvalues and three lead eigenvectors of the normalized Laplacian graph matrix for the Han depression network.

\begin{tabular}{cccccc}
\hline & Eigenvalue & Eigengap & \multicolumn{3}{c}{ Eigenvectors } \\
$k$ & $\lambda_{k}$ & $\lambda_{K}^{d}$ & $\mathbf{u}_{1}$ & $\mathbf{u}_{2}$ & $\mathbf{u}_{3}$ \\
\hline 1 & 0.000000 & & -0.123655 & -0.089596 & -0.135272 \\
2 & 0.351010 & 0.114655 & -0.123655 & 0.167580 & -0.012995 \\
3 & 0.465666 & 0.168739 & -0.123655 & 0.212885 & -0.034526 \\
4 & 0.634405 & 0.094340 & -0.123655 & 0.118713 & -0.014908 \\
5 & 0.728745 & 0.095914 & -0.123655 & 0.208821 & 0.007031 \\
6 & 0.824659 & 0.087227 & -0.123655 & -0.128337 & -0.083181 \\
7 & 0.911886 & 0.005848 & -0.123655 & 0.073023 & 0.025374 \\
8 & 0.917735 & 0.086178 & -0.123655 & -0.094594 & -0.080633 \\
9 & 1.003912 & 0.034640 & -0.123655 & -0.114313 & -0.081918 \\
10 & 1.038552 & 0.028874 & -0.123655 & -0.066315 & -0.074182 \\
11 & 1.067426 & 0.100654 & -0.123655 & 0.091590 & -0.081995 \\
12 & 1.168080 & 0.011602 & -0.123655 & 0.183010 & 0.034184 \\
13 & 1.179682 & 0.086197 & -0.123655 & 0.147287 & -0.024954 \\
14 & 1.265878 & 0.087154 & -0.123655 & 0.043342 & -0.083201 \\
15 & 1.353032 & 0.078109 & -0.123655 & -0.062875 & 0.484620 \\
16 & 1.431141 & 0.055221 & -0.123655 & -0.143029 & -0.063147 \\
17 & 1.486362 & 0.042661 & -0.123655 & -0.113256 & 0.236208 \\
18 & 1.529023 & 0.113783 & -0.123655 & -0.130745 & 0.056439 \\
19 & 1.642806 & & -0.123655 & -0.026453 & 0.288269 \\
\hline & & & & &
\end{tabular}


Table 2. The eigenvalues and four lead eigenvectors of the normalized Laplacian graph matrix for the Obama network.

\begin{tabular}{ccccccc}
\hline & Eigenvalue & Eigengap & \multicolumn{4}{c}{ Eigenvectors } \\
\cline { 4 - 7 }$k$ & $\lambda_{k}$ & $\lambda_{K}^{d}$ & -0.125848 & -0.056527 & 0.066447 & 0.203221 \\
\hline 1 & 0.000000 & & -0.125848 & 0.001137 & 0.025642 & -0.091254 \\
2 & 0.602133 & 0.105619 & -0.125848 & -0.013470 & -0.014170 & 0.129110 \\
3 & 0.707752 & 0.166750 & -0.125848 & -0.141460 & 0.069760 & -0.177129 \\
4 & 0.874502 & 0.189594 & -0.125848 & -0.153043 & 0.100122 & -0.107654 \\
5 & 1.064096 & 0.113360 & -0.125848 & -0.023113 & 0.066461 & 0.167682 \\
6 & 1.177456 & 0.080127 & -0.125848 & 0.284888 & 0.102135 & -0.073556 \\
7 & 1.257583 & 0.147878 & -0.125848 & 0.027185 & -0.248180 & -0.047212 \\
8 & 1.405461 & 0.036285 & -0.125848 & 0.234171 & 0.066826 & -0.053883 \\
9 & 1.441747 & 0.027524 & -0.125848 & -0.018674 & -0.263215 & -0.002554 \\
\hline 10 & 1.469270 & & & & $\mathbf{u}_{2}$ & \\
\hline
\end{tabular}


Table 3. Simulation 1 (equal cluster sizes, known $K$ ) results: The top panel numbers are average ARI values, the bottom panel numbers are the percentage of problems for which there was perfect recovery.

\begin{tabular}{|c|c|c|c|c|c|c|}
\hline & \multicolumn{2}{|c|}{ Walktrap Algorithm } & \multicolumn{2}{|c|}{ Spectral (Unnormalized) } & \multicolumn{2}{|c|}{ Spectral (Normalized) } \\
\hline & Ward's & $K$-means & $K$-means & $K$-median & $K$-means & $K$-median \\
\hline Overall & .9189 & .9264 & .8691 & .8695 & .9404 & .9415 \\
\hline$K=2$ & 1.0000 & 1.0000 & 1.0000 & 1.0000 & 1.0000 & 1.0000 \\
\hline$K=3$ & .9881 & .9895 & .9586 & .9569 & .9909 & .9909 \\
\hline$K=4$ & .9444 & .9590 & .9013 & .9009 & .9588 & .9604 \\
\hline$K=5$ & .8634 & .8763 & .7753 & .7758 & .9235 & .9169 \\
\hline$K=6$ & .7988 & .8072 & .7105 & .7139 & .8286 & .8392 \\
\hline Cohesion -0.9 & .9753 & .9804 & .9487 & .9534 & .9885 & .9888 \\
\hline Cohesion -0.8 & .9263 & .9330 & .8811 & .8784 & .9439 & .9416 \\
\hline Cohesion -0.7 & .8553 & .8659 & .7776 & .7767 & .8888 & .8941 \\
\hline Overlap -0.1 & .9612 & .9620 & .9388 & .9393 & .9654 & .9673 \\
\hline Overlap -0.2 & .9264 & .9381 & .8703 & .8757 & .9519 & .9524 \\
\hline Overlap - 0.3 & .8692 & .8791 & .7984 & .7935 & .9039 & .9048 \\
\hline Overall & 68.2 & 71.3 & 62.0 & 62.2 & 74.4 & 74.7 \\
\hline$K=2$ & 100.0 & 100.0 & 100.0 & 100.0 & 100.0 & 100.0 \\
\hline$K=3$ & 93.3 & 94.4 & 88.9 & 88.9 & 95.6 & 95.6 \\
\hline$K=4$ & 67.8 & 75.6 & 61.1 & 60.0 & 76.7 & 77.8 \\
\hline$K=5$ & 52.2 & 53.3 & 36.7 & 36.7 & 65.6 & 62.2 \\
\hline$K=6$ & 27.8 & 33.3 & 23.3 & 25.6 & 34.4 & 37.8 \\
\hline Cohesion -0.9 & 85.3 & 88.0 & 80.0 & 82.0 & 91.3 & 92.0 \\
\hline Cohesion -0.8 & 66.7 & 68.7 & 62.0 & 60.7 & 72.0 & 70.7 \\
\hline Cohesion -0.7 & 52.7 & 57.3 & 44.0 & 44.0 & 60.0 & 61.3 \\
\hline Overlap - 0.1 & 81.3 & 82.0 & 77.3 & 76.7 & 83.3 & 82.7 \\
\hline Overlap -0.2 & 67.3 & 74.0 & 59.3 & 60.0 & 74.7 & 75.3 \\
\hline Overlap - 0.3 & 56.0 & 58.0 & 49.3 & 50.0 & 65.3 & 66.0 \\
\hline
\end{tabular}


Table 4. Simulation 2 (unequal cluster sizes, known $K$ ) results: The top panel numbers are average ARI values, the bottom panel numbers are the percentage of problems for which there was perfect recovery.

\begin{tabular}{|c|c|c|c|c|c|c|}
\hline & \multicolumn{2}{|c|}{ Walktrap Algorithm } & \multicolumn{2}{|c|}{ Spectral (Unnormalized) } & \multicolumn{2}{|c|}{ Spectral (Normalized) } \\
\hline & Ward's & $K$-means & $K$-means & $K$-median & $K$-means & $K$-median \\
\hline Overall & .9340 & .9369 & .8947 & .8765 & .9489 & .9495 \\
\hline$K=2$ & .9957 & .9957 & .9315 & .8886 & 1.0000 & .9957 \\
\hline$K=3$ & .9882 & .9860 & .9881 & .9722 & .9833 & .9834 \\
\hline$K=4$ & .9593 & .9623 & .9405 & .9314 & .9622 & .9659 \\
\hline$K=5$ & .8968 & .9004 & .8553 & .8404 & .9105 & .9173 \\
\hline$K=6$ & .8299 & .8401 & .7582 & .7497 & .8884 & .8854 \\
\hline Cohesion -0.9 & .9778 & .9797 & .9539 & .9331 & .9895 & .9883 \\
\hline Cohesion -0.8 & .9411 & .9423 & .8958 & .8814 & .9612 & .9569 \\
\hline Cohesion -0.7 & .8830 & .8886 & .8344 & .8149 & .8959 & .9035 \\
\hline Overlap -0.1 & .9772 & .9802 & .9580 & .9425 & .9801 & .9832 \\
\hline Overlap -0.2 & .9396 & .9389 & .8830 & .8661 & .9479 & .9482 \\
\hline Overlap - 0.3 & .8852 & .8916 & .8432 & .8208 & .9187 & .9173 \\
\hline Overall & 71.3 & 71.8 & 62.4 & 53.1 & 74.0 & 73.6 \\
\hline$K=2$ & 98.9 & 98.9 & 82.2 & 71.1 & 100.0 & 98.9 \\
\hline$K=3$ & 94.4 & 91.1 & 88.9 & 71.1 & 92.2 & 92.2 \\
\hline$K=4$ & 73.3 & 74.4 & 67.8 & 57.8 & 75.6 & 74.4 \\
\hline$K=5$ & 54.4 & 57.8 & 47.8 & 44.4 & 58.9 & 60.0 \\
\hline$K=6$ & 35.6 & 36.7 & 25.6 & 21.1 & 43.3 & 42.2 \\
\hline Cohesion -0.9 & 86.0 & 87.3 & 74.7 & 62.7 & 91.3 & 90.0 \\
\hline Cohesion -0.8 & 69.3 & 70.0 & 61.3 & 51.3 & 74.0 & 73.3 \\
\hline Cohesion -0.7 & 58.7 & 58.0 & 51.3 & 45.3 & 56.7 & 57.3 \\
\hline Overlap - 0.1 & 88.0 & 88.0 & 80.7 & 72.0 & 86.0 & 88.0 \\
\hline Overlap -0.2 & 70.0 & 70.0 & 57.3 & 46.0 & 76.0 & 73.3 \\
\hline Overlap - 0.3 & 56.0 & 57.3 & 49.3 & 41.3 & 60.0 & 59.3 \\
\hline
\end{tabular}


Table 5. Simulation 3 (equal cluster sizes, unknown $K$ ) results: The top panel numbers are average ARI values, the bottom panel numbers are the percentage of problems for which there was perfect recovery.

\begin{tabular}{|c|c|c|c|c|c|c|}
\hline & \multicolumn{2}{|c|}{ Walktrap Algorithm } & \multicolumn{2}{|c|}{ Spectral (Unnormalized) } & \multicolumn{2}{|c|}{ Spectral (Normalized) } \\
\hline & Ward's & $K$-means & $K$-means & $K$-median & $K$-means & $K$-median \\
\hline Overall & .8635 & .8679 & .8458 & .8471 & .9133 & .9128 \\
\hline$K=2$ & 1.0000 & 1.0000 & .9973 & .9973 & 1.0000 & 1.0000 \\
\hline$K=3$ & .9819 & .9833 & .9563 & .9559 & .9893 & .9893 \\
\hline$K=4$ & .9050 & .9154 & .8810 & .8824 & .9569 & .9573 \\
\hline$K=5$ & .7384 & .7434 & .7402 & .7411 & .8484 & .8390 \\
\hline$K=6$ & .6923 & .6976 & .6541 & .6589 & .7721 & .7782 \\
\hline Cohesion -0.9 & .9450 & .9474 & .9289 & .9261 & .9763 & .9771 \\
\hline Cohesion -0.8 & .8643 & .8691 & .8584 & .8618 & .9120 & .9111 \\
\hline Cohesion -0.7 & .7813 & .7873 & .7501 & .7535 & .8516 & .8501 \\
\hline Overlap -0.1 & .9471 & .9480 & .9165 & .9172 & .9631 & .9615 \\
\hline Overlap -0.2 & .8730 & .8746 & .8699 & .8746 & .9414 & .9435 \\
\hline Overlap - 0.3 & .7705 & .7812 & .7509 & .7495 & .8355 & .8333 \\
\hline Overall & 62.9 & 64.7 & 49.8 & 49.3 & 71.8 & 72.2 \\
\hline$K=2$ & 100.0 & 100.0 & 96.7 & 96.7 & 100.0 & 100.0 \\
\hline$K=3$ & 91.1 & 92.2 & 75.6 & 75.6 & 93.3 & 93.3 \\
\hline$K=4$ & 57.8 & 63.3 & 43.3 & 42.2 & 75.6 & 76.7 \\
\hline$K=5$ & 40.0 & 41.1 & 17.8 & 16.7 & 58.9 & 56.7 \\
\hline$K=6$ & 25.6 & 26.7 & 15.6 & 15.6 & 31.1 & 34.4 \\
\hline Cohesion -0.9 & 79.3 & 81.3 & 66.0 & 66.0 & 90.0 & 90.0 \\
\hline Cohesion -0.8 & 62.7 & 63.3 & 48.0 & 46.7 & 67.3 & 67.3 \\
\hline Cohesion -0.7 & 46.7 & 49.3 & 35.3 & 35.3 & 58.0 & 59.3 \\
\hline Overlap -0.1 & 78.7 & 79.3 & 62.7 & 62.0 & 82.0 & 80.7 \\
\hline Overlap -0.2 & 60.7 & 62.7 & 44.7 & 44.7 & 72.0 & 74.0 \\
\hline Overlap - 0.3 & 49.3 & 52.0 & 42.0 & 41.3 & 61.3 & 62.0 \\
\hline
\end{tabular}


Table 6. Simulation 3 (equal cluster size, unknown $K$ ) results: The percentage of test problems for which the true number of clusters $\left(K^{*}\right)$ was underestimated $\left(K<K^{*}\right)$, correctly estimated $(K$ $\left.=K^{*}\right)$, and overestimated $\left(K>K^{*}\right)$ using the ratio rule of Pons and Latapy (2006) for the walktrap method and the eigengap heuristic for spectral clustering for both the unnormalized and normalized Laplacian matrices.

\begin{tabular}{|c|c|c|c|c|c|c|c|c|c|}
\hline & \multicolumn{3}{|c|}{ Walktrap - ratio rule } & \multicolumn{3}{|c|}{ Spectral (Unnormalized) - eigengap } & \multicolumn{3}{|c|}{ Spectral (normalized) - eigengap } \\
\hline & $K<K^{*}$ & $K=K^{*}$ & $K>K^{*}$ & $K<K^{*}$ & $K=K^{*}$ & $K>K^{*}$ & $K<K^{*}$ & $K=K^{*}$ & $K>K^{*}$ \\
\hline Overall & 18.2 & 74.4 & 7.3 & 12.9 & 58.2 & 28.9 & 9.3 & 85.3 & 5.3 \\
\hline$K=2$ & 0.0 & 100.0 & 0.0 & 0.0 & 96.7 & 3.3 & 0.0 & 100.0 & 0.0 \\
\hline$K=3$ & 1.1 & 97.8 & 1.1 & 3.3 & 77.8 & 18.9 & 0.0 & 95.6 & 4.4 \\
\hline$K=4$ & 8.9 & 77.8 & 13.3 & 4.4 & 54.4 & 41.1 & 3.3 & 95.6 & 1.1 \\
\hline$K=5$ & 35.6 & 52.2 & 12.2 & 25.6 & 24.4 & 50.0 & 17.8 & 80.0 & 2.2 \\
\hline$K=6$ & 45.6 & 44.4 & 10.0 & 31.1 & 37.8 & 31.1 & 25.6 & 55.6 & 18.9 \\
\hline Cohesion -0.9 & 10.7 & 86.7 & 2.7 & 8.7 & 72.0 & 19.3 & 3.3 & 95.3 & 1.3 \\
\hline Cohesion -0.8 & 18.7 & 73.3 & 8.0 & 12.0 & 57.3 & 30.7 & 10.7 & 84.0 & 5.3 \\
\hline Cohesion -0.7 & 25.3 & 63.3 & 11.3 & 18.0 & 45.3 & 36.7 & 14.0 & 76.7 & 9.3 \\
\hline Overlap - 0.1 & 5.3 & 87.3 & 7.3 & 5.3 & 68.7 & 26.0 & 3.3 & 92.0 & 4.7 \\
\hline Overlap -0.2 & 16.7 & 73.3 & 10.0 & 10.0 & 52.7 & 37.3 & 6.0 & 87.3 & 6.7 \\
\hline Overlap - 0.3 & 32.7 & 62.7 & 4.7 & 23.3 & 53.3 & 23.3 & 18.7 & 76.7 & 4.7 \\
\hline
\end{tabular}


Table 7. Simulation 4 (unequal cluster size, unknown $K$ ) results: The top panel numbers are average ARI values, the bottom panel numbers are the percentage of problems for which there was perfect recovery.

\begin{tabular}{|c|c|c|c|c|c|c|}
\hline & \multicolumn{2}{|c|}{ Walktrap Algorithm } & \multicolumn{2}{|c|}{ Spectral (Unnormalized) } & \multicolumn{2}{|c|}{ Spectral (Normalized) } \\
\hline & Ward's & $K$-means & $K$-means & $K$-median & $K$-means & $K$-median \\
\hline Overall & .8708 & .8719 & .8636 & .8551 & .8745 & .8729 \\
\hline$K=2$ & .9661 & .9661 & .9759 & .9716 & .9837 & .9795 \\
\hline$K=3$ & .9352 & .9365 & .9786 & .9745 & .8863 & .8852 \\
\hline$K=4$ & .9181 & .9205 & .9008 & .8956 & .9100 & .9090 \\
\hline$K=5$ & .8227 & .8265 & .7659 & .7530 & .8206 & .8239 \\
\hline$K=6$ & .7118 & .7099 & .6970 & .6808 & .7718 & .7670 \\
\hline Cohesion -0.9 & .8952 & .8964 & .9207 & .9132 & .9211 & .9192 \\
\hline Cohesion -0.8 & .8915 & .8923 & .8695 & .8639 & .8932 & .8858 \\
\hline Cohesion -0.7 & .8257 & .8270 & .8007 & .7882 & .8092 & .8137 \\
\hline Overlap -0.1 & .9589 & .9605 & .9579 & .9537 & .9502 & .9505 \\
\hline Overlap -0.2 & .8730 & .8719 & .8728 & .8594 & .8757 & .8758 \\
\hline Overlap - 0.3 & .7805 & .7833 & .7602 & .7522 & .7976 & .7924 \\
\hline Overall & 50.2 & 51.1 & 26.0 & 22.9 & 48.4 & 47.8 \\
\hline$K=2$ & 82.2 & 82.2 & 1.1 & 0.0 & 97.8 & 96.7 \\
\hline$K=3$ & 54.4 & 54.4 & 38.9 & 33.3 & 30.0 & 28.9 \\
\hline$K=4$ & 48.9 & 51.1 & 42.2 & 37.8 & 42.2 & 41.1 \\
\hline$K=5$ & 41.1 & 42.2 & 28.9 & 26.7 & 37.8 & 37.8 \\
\hline$K=6$ & 24.4 & 25.6 & 18.9 & 16.7 & 34.4 & 34.4 \\
\hline Cohesion -0.9 & 57.3 & 58.0 & 28.0 & 24.0 & 55.3 & 55.3 \\
\hline Cohesion -0.8 & 50.7 & 51.3 & 23.3 & 21.3 & 51.3 & 49.3 \\
\hline Cohesion -0.7 & 42.7 & 44.0 & 26.7 & 23.3 & 38.7 & 38.7 \\
\hline Overlap - 0.1 & 71.3 & 72.0 & 42.0 & 38.0 & 68.7 & 69.3 \\
\hline Overlap -0.2 & 44.7 & 45.3 & 22.0 & 18.0 & 42.0 & 40.7 \\
\hline Overlap - 0.3 & 34.7 & 36.0 & 14.0 & 12.7 & 34.7 & 33.3 \\
\hline
\end{tabular}


Table 8. Simulation 4 (unequal cluster size, unknown $K$ ) results: The percentage of test problems for which the true number of clusters $\left(K^{*}\right)$ was underestimated $\left(K<K^{*}\right)$, correctly estimated $(K$ $\left.=K^{*}\right)$, and overestimated $\left(K>K^{*}\right)$ using the ratio rule of Pons and Latapy (2006) for the walktrap method and the eigengap heuristic for spectral clustering for both the unnormalized and normalized Laplacian matrices.

\begin{tabular}{|c|c|c|c|c|c|c|c|c|c|}
\hline & \multicolumn{3}{|c|}{ Walktrap - ratio rule } & \multicolumn{3}{|c|}{ Spectral (Unnormalized) - eigengap } & \multicolumn{3}{|c|}{ Spectral (normalized) - eigengap } \\
\hline & $K<K^{*}$ & $K=K^{*}$ & $K>K^{*}$ & $K<K^{*}$ & $K=K^{*}$ & $K>K^{*}$ & $K<K^{*}$ & $K=K^{*}$ & $K>K^{*}$ \\
\hline Overall & 33.3 & 56.4 & 10.2 & 16.2 & 34.4 & 49.3 & 42.7 & 54.4 & 2.9 \\
\hline$K=2$ & 0.0 & 83.3 & 16.7 & 0.0 & 3.3 & 96.7 & 0.0 & 97.8 & 2.2 \\
\hline$K=3$ & 38.9 & 54.4 & 6.7 & 3.3 & 43.3 & 53.3 & 70.0 & 30.0 & 0.0 \\
\hline$K=4$ & 36.7 & 56.7 & 6.7 & 14.4 & 55.6 & 30.0 & 51.1 & 47.8 & 1.1 \\
\hline$K=5$ & 38.9 & 52.2 & 8.9 & 26.7 & 41.1 & 32.2 & 50.0 & 46.7 & 3.3 \\
\hline$K=6$ & 52.2 & 35.6 & 12.2 & 36.7 & 28.9 & 34.4 & 42.2 & 50.0 & 7.8 \\
\hline Cohesion -0.9 & 33.3 & 58.7 & 8.0 & 9.3 & 36.7 & 54.0 & 42.0 & 56.7 & 1.3 \\
\hline Cohesion -0.8 & 28.7 & 59.3 & 12.0 & 18.0 & 30.0 & 52.0 & 40.7 & 56.7 & 2.7 \\
\hline Cohesion -0.7 & 38.0 & 51.3 & 10.7 & 21.3 & 36.7 & 42.0 & 45.3 & 50.0 & 4.7 \\
\hline Overlap - 0.1 & 8.7 & 76.7 & 14.7 & 4.7 & 46.7 & 48.7 & 20.7 & 76.0 & 3.3 \\
\hline Overlap -0.2 & 36.0 & 52.0 & 12.0 & 15.3 & 30.7 & 54.0 & 47.3 & 50.0 & 2.7 \\
\hline Overlap -0.3 & 55.3 & 40.7 & 4.0 & 28.7 & 26.0 & 45.3 & 60.0 & 37.3 & 2.7 \\
\hline
\end{tabular}




$\downarrow$ interest
worthlessness
suicidality
$\downarrow$ self-esteem
$\downarrow$ confidence
hopelessness
helplessness
$\Delta$ weight, appetite
$\Delta$ sleep
$\downarrow$ motor movement
fatigue
difficulty concentrating
distinct quality of depressed mood
feel worse in AM
$\downarrow$ libido
unreactive mood
irritable/angry
crying
nervousness

\begin{tabular}{|c|c|c|c|c|c|c|c|c|c|c|c|c|c|c|c|c|c|c|}
\hline 2 & 7 & 9 & 10 & 11 & 17 & 19 & 3 & 4 & 5 & 6 & 8 & 12 & 13 & 14 & 15 & 16 & 18 & 20 \\
\hline- & .00 & .00 & .00 & .00 & .55 & .00 & .00 & .00 & .00 & .00 & .00 & .00 & .00 & .00 & .46 & .00 & .00 & .00 \\
\hline .00 & - & .74 & 1.23 & .46 & .88 & .46 & .00 & .00 & .20 & .00 & .32 & .00 & .00 & .00 & .21 & .00 & .13 & 00 \\
\hline .00 & .74 & - & .00 & .13 & 1.71 & .00 & .18 & .28 & .18 & .00 & .00 & .14 & .00 & .00 & .30 & .00 & .26 & .00 \\
\hline .00 & 1.23 & .00 & - & 1.52 & .39 & .44 & .00 & .00 & .16 & .00 & .00 & .00 & .08 & .22 & .00 & .00 & .00 & \\
\hline .00 & .46 & .13 & 1.52 & - & .37 & .62 & .00 & .00 & .37 & .00 & .81 & .00 & .00 & .21 & .60 & .00 & .00 & 00 \\
\hline .55 & .88 & 1.71 & .39 & .37 & - & 1.26 & .00 & .00 & .00 & .00 & .00 & .00 & .00 & .00 & .44 & .00 & .08 & \\
\hline .00 & .46 & .00 & .44 & .62 & 1.26 & - & .00 & .00 & .24 & .00 & .00 & .00 & .00 & .13 & .00 & .00 & .70 & \\
\hline .00 & .00 & .18 & .00 & .00 & .00 & .00 & - & .38 & .66 & .55 & .00 & .00 & .00 & .22 & 37 & .00 & .22 & \\
\hline .00 & .00 & .28 & .00 & .00 & .00 & .00 & .38 & - & .37 & .76 & .00 & .00 & .00 & .82 & .00 & .00 & .00 & \\
\hline .00 & .20 & .18 & .16 & .37 & .00 & .24 & .66 & .37 & - & .70 & 1.26 & .41 & .33 & .25 & .66 & .00 & .00 & \\
\hline .00 & .00 & .00 & .00 & .00 & .00 & .00 & .55 & .76 & .70 & - & .50 & .00 & .24 & .08 & .18 & .00 & .00 & \\
\hline .00 & .32 & .00 & .00 & .81 & .00 & .00 & .00 & .00 & 1.26 & .50 & - & .00 & .00 & .37 & .58 & .00 & .00 & \\
\hline .00 & .00 & .14 & .00 & .00 & .00 & .00 & .00 & .00 & .41 & .00 & .00 & - & .00 & .00 & .17 & .00 & .00 & \\
\hline .00 & .00 & .00 & .08 & .00 & .00 & .00 & .00 & .00 & .33 & .24 & .00 & .00 & - & .47 & .00 & .09 & .00 & \\
\hline .00 & .00 & .00 & .22 & .21 & .00 & .13 & .22 & .82 & .25 & .08 & .37 & .00 & .47 & - & .63 & .00 & .00 & \\
\hline .46 & .21 & .30 & .00 & .60 & .44 & .00 & .37 & .00 & .66 & .18 & .58 & .17 & .00 & .63 & - & .00 & .00 & \\
\hline .00 & .00 & .00 & .00 & .00 & .00 & .00 & .00 & .00 & .00 & .00 & .00 & .00 & .09 & .00 & .00 & - & .49 & 1.0 \\
\hline .00 & .13 & .26 & .00 & .00 & .08 & .70 & .22 & .00 & .00 & .00 & .00 & .00 & .00 & .00 & .00 & .49 & - & \\
\hline .00 & .00 & .00 & .13 & .00 & .31 & .61 & .00 & .00 & .27 & .18 & .77 & .00 & .00 & .14 & .00 & 1.07 & .00 & \\
\hline
\end{tabular}

Figure 1. A partition of the adjacency matrix for the three-cluster spectral clustering solution for the Han depression network. 


\begin{tabular}{|c|c|c|c|c|c|c|c|c|c|c|c|}
\hline & & 1 & 3 & 6 & 2 & 4 & 5 & 8 & 10 & 7 & 9 \\
\hline & & Mor & Car & Hns & Led & Kno & Int & Hop & Prd & Ang & Afr \\
\hline Moral & 1 & -- & 1.23 & 1.76 & .38 & .49 & 1.13 & .19 & .52 & .22 & .42 \\
\hline Caring & 3 & 1.23 & --- & 1.38 & .801 & .65 & .68 & .78 & .86 & .49 & .48 \\
\hline Honest & 6 & 1.76 & 1.38 & $\cdots$ & .93 & .67 & .86 & .34 & .42 & .36 & .73 \\
\hline Leadership & 2 & .38 & .80 & .93 & لـ--ב. & 1.38 & .58 & .33 & .82 & .84 & .46 \\
\hline Knowedgeable & 4 & .49 & .65 & .67 & 1.38 & --- & 2.66 & .56 & .39 & .00 & .23 \\
\hline Intelligent & 5 & 1.13 & .68 & .86 & .58 & 2.66 & -- & .31 & .25 & .00 & .24 \\
\hline Hopeful & 8 & .19 & .78 & .34 & .33 & .56 & .31 & --- & 2.32 & .28 & .78 \\
\hline Proud & 10 & .52 & .86 & .42 & .82 & .39 & .25[ & 2.32 & -- & .00 & .16 \\
\hline Angry & 7 & .22 & .49 & .36 & .84 & .00 & .00 & .28 & .00 & -- & 2.21 \\
\hline Afraid & 9 & .42 & .48 & .73 & .46 & .23 & .24 & .78 & .16 & 2.21 & -- \\
\hline
\end{tabular}

Figure 2. A partition of the adjacency matrix for the four-cluster spectral clustering solution for the Obama network. The dashed lines show the slightly different four-cluster partition obtained via the walktrap algorithm, whereby 'Leadership' is placed with 'Moral', 'Caring', and 'Honest', rather than 'Knowledgeable' and 'Intelligent'. 